\title{
Patient-Reported Factors Facilitating Participation in Prevention of Mother to Child Transmission of HIV Programs in Kara, Togo, West Africa
}

\author{
Eméfah C. Loccoh1, Deladem Azouma2,3, Kevin Fiori Jr.4, Sesso Gbeleou, \\ Jennifer Schechter ${ }^{4}$, Lisa R. Hirschhorn $6,7^{*}$ \\ ${ }^{1}$ The University of Michigan, Ann Arbor, MI, USA \\ ${ }^{2}$ The University of Lomé, Lomé, Togo \\ ${ }^{3}$ The University of Kara, Kara, Togo \\ ${ }^{4}$ Hope Through Health, Medway, MA, USA \\ ${ }^{5} \mathrm{~L}^{\prime}$ Association Espoir pour Demain, Kara, Togo \\ ${ }^{6}$ Harvard Medical School, Boston, MA, USA \\ ${ }^{7}$ Ariadne Labs, Boston, MA, USA \\ Email: eloccoh@umich.edu, adkazoum@yahoo.fr, kfiori@hthglobal.org, Sessogbeleou@yahoo.fr, \\ jschechter@hthglobal.org,
}

Received 25 September 2014; revised 20 October 2014; accepted 23 November 2014

Copyright (C) 2014 by authors and Scientific Research Publishing Inc.

This work is licensed under the Creative Commons Attribution International License (CC BY).

http://creativecommons.org/licenses/by/4.0/

(c) (i) Open Access

\section{Abstract}

Background: Despite efforts to expand prevention of mother-to-child transmission (PMTCT) of HIV in resource-limited settings, only $53 \%$ of women in sub-Saharan Africa receive adequate PMTCT services. Understanding factors that enable successful program completion are crucial to improving adherence rates to PMTCT programs in these settings. Using a positive deviance approach, we explored patient and provider's perspectives on factors enabling women to successfully access and adhere to PMTCT services to identify areas of program improvement and further reduce HIV transmission. Methods: Semi-structured interviews were conducted with 11 health care workers (HCWs) at two health centers in Kara, Togo and 34 women who had successfully completed the PMTCT program at those facilities. Women were identified using purposive sampling and content theme analysis was used to analyze the data. Results: Knowledge and belief in the potential to give birth to an HIV-negative child, supported by facilitating factors on the indi-

\footnotetext{
"Corresponding author.
}

How to cite this paper: Loccoh, E.C., Azouma, D., Fiori Jr., K., Gbeleou, S., Schechter, J. and Hirschhorn, L.R. (2014) Patient-Reported Factors Facilitating Participation in Prevention of Mother to Child Transmission of HIV Programs in Kara, Togo, West Africa. World Journal of AIDS, 4, 446-457. http://dx.doi.org/10.4236/wja.2014.44053 
vidual and community levels, was the cornerstone to women's PMTCT program success. Effective program education and peer-to-peer interactions fostered the participants' knowledge and belief in PMTCT, with the resulting internal motivation driving continued participation and adherence. The welcoming environment, availability of peer support groups and financial assistance from the health facility, further facilitated success, with women using this facility-based community to seek advice and support from HCWs and peers. Financial, physical, and emotional support from relatives was also important in ensuring unhindered access. Finally, the faith-based communities' acceptance and support for HIV-positive women further encouraged participants to adhere to the program. Conclusion: Understanding and believing in the ability to have an HIV-negative baby was the most important facilitator for PMTCT program adherence. This led to internal motivation that was continually reinforced through facility and community supportive environments focused on addressing barriers. Efforts to improve PMTCT success should focus on all of these factors through a patient-centered approach.

\section{Keywords}

Maternal Health, Program Adherence, AIDS, Mother-to-Child Transmission, HIV Positive Women

\section{Introduction}

With no intervention, $20 \%$ - 50\% of infants born to HIV positive mothers will become infected during pregnancy, delivery, and through breastfeeding [1]. With the initiation of effective prevention of mother-to-child transmission (PMTCT) of HIV programs, this risk can be reduced to under 2\% [1]. However, many barriers still exist for women to complete the full set of interventions needed for effective PMTCT programs [2]. Therefore, it is important to understand areas that can be strengthened to create delivery models able to increase adherence throughout the entire risk period [3].

In 2011, 30\% of pregnant, HIV positive women in Togo participated in PMTCT programs, and 48\% of these women were lost to follow-up [4]. Factors associated with poor uptake of and adherence to PMTCT in sub-Saharan Africa includes lack of partner support, fear of stigma and discrimination, and social and cultural taboos [5] [6]. Less is known about factors associated with program success. One method to identify why some women succeed where others fail is by studying these individuals through a positive deviance approach [7].

Hope Through Health (HTH), a US-based not-for-profit organization, partners with Association Espoir pour Demain (AED-Lidaw), a community-based association of HIV positive individuals living in Togo, to deliver facility and community-based health interventions. The partnership supports a PMTCT program, delivered at the AED-Lidaw/HTH health center, which includes socioeconomic and psychosocial support in addition to medical care provided according to national guidelines [8]. The Central Hospitalier Universitaire-Kara (CHU-Kara) is a public referral hospital located in the northern region of Togo. A PMTCT program providing medical care according to national guidelines is offered through the Obstetrics and Gynecology Department; however, at the time of study, the program did not offer socioeconomic or psychosocial support to participants in conjunction with the program.

We report on the results of a qualitative evaluation of successful PMTCT participants and PMTCT health care workers (HCWs), at AED-Lidaw/HTH and CHU-Kara, to identify factors that facilitated the women’s participation and adherence to program protocols. These findings can provide valuable insights on how to strengthen programs to increase participation and adherence for current and future participants in other PMTCT programs in Togo and similar settings in the region.

\section{Methods}

\subsection{Study Design}

We utilized an exploratory qualitative study design using semi-structured interviews and content theme analysis, focusing on women who successfully adhered to the PMTCT program and HCWs employed in the facilities where these women received care. 


\subsection{Settings}

Interviews were conducted in the PMTCT clinics at two settings: AED-Lidaw/HTH health center, located in Kara, Togo, which serves over 1600 HIV positive individuals and CHU-Kara, the largest public hospital in the Kara region, located approximately 4 kilometers from AED-Lidaw/HTH health center. Both sites have PMTCT clinics, with AED-Lidaw/HTH offering additional resources to support participant adherence. At the time of interview, over 40 women were enrolled in the PMTCT program at AED-Lidaw/HTH, and over 100 women at CHU-Kara; 40 of these women were identified as having recently successfully completed the PMTCT protocol at least through the delivery process. PMTCT protocols at both sites reflected those of Togo's Ministry of Health, which were based on the World Health Organization guidelines at the time of study using antiretroviral prophylaxis Option A [9] [10].

\subsection{Participants and Sampling Frame}

Participants were chosen through purposive sampling of women who successfully completed at least the prenatal and labor and delivery PMTCT protocols at one of the two facilities between April 1, 2011 and October 1, 2012. Successful completion of post-natal guidelines was not an inclusion criterion as many women were still in the process of completing these guidelines. Women were identified by PMTCT program HCWs from both sites and contacted via phone to arrange interview dates and times. Participants were recruited between JanuaryMarch 2013 with interviews conducted between April and May 2013. Thirty-four women were interviewed (25 from AED, 9 from CHU). Participants were provided transportation reimbursement and a small stipend equivalent to one United States dollar as an incentive to participate in the study.

HCWs were eligible for inclusion in the study if they were working in the PMTCT program at either site. Purposive sampling was used to identify eleven HCWS who served in either management roles (i.e. Director, Department Director, etc.) or as direct care providers (i.e. doctor, psychologist, mid-wife, etc.) in the PMTCT programs at one of the sites.

\subsection{Data Collection}

Two semi-structured interview guides, one for HCWs and one for PMTCT participants, were developed in French with translation of patient guide into Kabiyé, the local language, and back into French to ensure accuracy. Both guides were piloted and questions refined, as needed.

In depth, face-to-face interviews with the women were conducted in a private room within CHU-Kara, regardless of where participants had received PMTCT care. The first author (LE), and a study nurse, with experience in HIV treatment and prevention, conducted the interviews in French or Kabiyé based on participants' preference. Interviews were recorded, and lasted between 30 - 60 minutes.

Areas of focus included experiences pre-, peri-, and post-delivery, related to PMTCT and adherence experiences. The first author reviewed interviews with two other authors (SJ, HL) to summarize dialogues and suggest changes for future interviews (emergent design) [11]. Some questions were reworded for enhanced clarity during future interviews.

Interviews were conducted one-on-one with the HCWs in their offices either at AED-Lidaw/HTH or CHUKara. One author (LE) conducted the interviews in French. The interviews were recorded and lasted between 30 60 minutes.

\subsection{Data Management and Analysis}

Interview recordings were transcribed and interviews conducted in Kabiyé were translated into French. Transcripts were compared with the audio recording to ensure accuracy of the transcription.

Data were analyzed using content theme analysis [12]. Table 1 provides an example of original, translated data with main themes and sub-themes. The first author coded and analyzed the data with a random sample of 10 interview transcripts reviewed by a second author to confirm accuracy.

\subsection{Ethical Considerations}

The Institutional Review Board (IRB) of Togo's Ministry of Health approved the main study protocol. All 
participants were interviewed after they were given an explanation of the study and signed a written consent form. All agreed to be audio-recorded.

\section{Results}

\subsection{Study Population Characteristics}

We interviewed 34 HIV positive women-twenty-five from the AED-Lidaw/HTH PMTCT program, nine from CHU-Kara who had successfully completed the PMTCT program. These women were between 23 to 40 years of age, (mean age of 31.9 years), with 65\% married (Table 2). Close to two thirds of participants had a primary school education, and a similar number (80\%) knew their HIV status prior to their pregnancy and were already taking antiretroviral (ART) medications at entry into the PMTCT program.

At the time of the interview, all of the women's infants had tested negative for HIV at the one-month pediatric follow-up. Additionally, all women were continuing to adhere to PMTCT post-delivery guidelines including clinical visits for themselves and their infants and continuing ART.

Six HCWs were interviewed from CHU-Kara and five from AED-Lidaw/HTH. Participants represented four clinical areas: pediatrics, obstetrics, PMTCT education, and overall HIV/AIDS program management (Table 3). HCWs included doctors (2), nurses (2), medical assistants (2) and one each of the following: community health worker, director, midwife, PMTCT program coordinator, and psychologist.

\subsection{Interview Results}

Factors were identified at the individual level (ensuring program knowledge, strengthening belief, fostering internal motivation) and community level (health facility, peer support, relatives, and faith-based). Factors at both levels reinforced women's internal motivation, which led to their PMTCT program success (Figure 1).

Table 1. Example of original data with main themes and sub-themes.

\begin{tabular}{l} 
Original Data \\
\hline $\begin{array}{l}\text { In the beginning it is not easy and a lot of [women] do not } \\
\text { accept [the PMTCT program]. But [those who do accept] } \\
\text { have understood that }\end{array}$ \\
$\begin{array}{lll}\text { 1) HIV is a disease like others and } & \text { 1) Health System } & \text { 1) Program Explanation } \\
\text { 2) for the health of their babies, they adhere. } & \text { 2) Individual } & \text { 2) Internal Motivation }\end{array}$
\end{tabular}

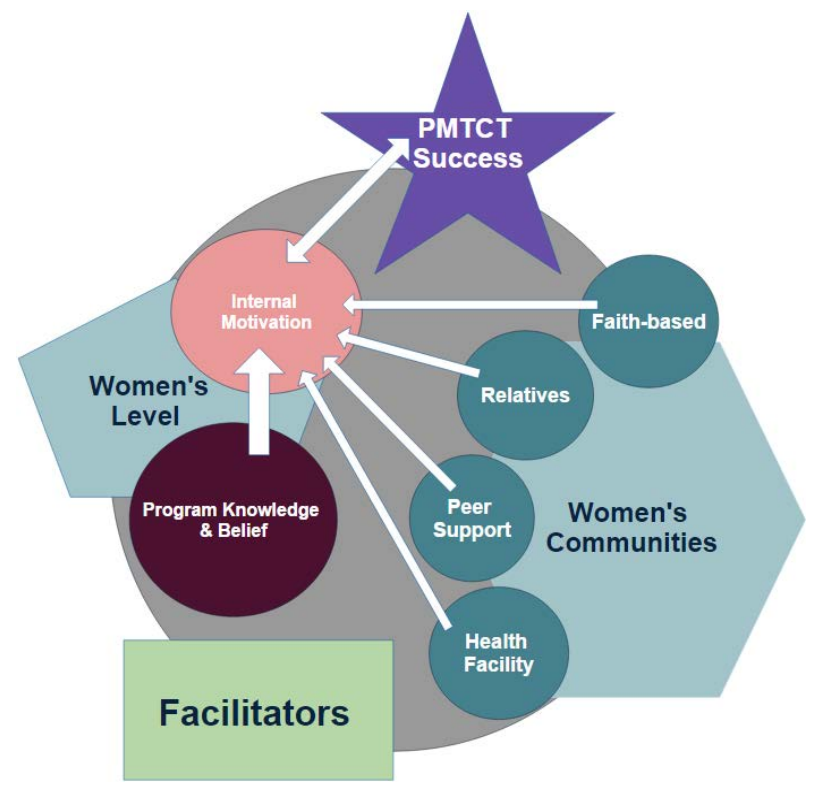

Figure 1. Conceptual model of facilitating factors. 
Table 2. Prevention of Mother to Child Transmission (PMTCT) of HIV participant demographics.

\begin{tabular}{lc}
\hline \multicolumn{1}{c}{ Participants } \\
Mean age (range) & 31.9 years \\
& $(23-40$ years) \\
Marital status & \\
- Married & $79 \%(27)$ \\
- Separated/Divorced & $18 \%(6)$ \\
Education & $3 \%(1)$ \\
- Primary & \\
- Secondary/Vocational Training & $70 \%(24)$ \\
HIV status known pre-pregnancy & $18 \%(6)$ \\
Ease getting to center & $80 \%(27)$ \\
- Slightly to very difficult & \\
Delivered at hospital & $76 \%(26)$ \\
\hline
\end{tabular}

Table 3. HCW participant demographics.

\begin{tabular}{|c|c|}
\hline HCW demographics & $\mathrm{N}$ \\
\hline \multicolumn{2}{|l|}{ Facility } \\
\hline - CHU-Kara & $6(55 \%)$ \\
\hline - HTH/AED/Lidaw & $5(45 \%)$ \\
\hline \multicolumn{2}{|l|}{ Domains } \\
\hline - Obstetrics \& Gynecology & 5 \\
\hline • Other PMTCT Program Staff & 3 \\
\hline - HIV/AIDS Program Management & 2 \\
\hline - Pediatrics & 1 \\
\hline \multicolumn{2}{|l|}{ Cadres } \\
\hline - Doctors & 2 \\
\hline - Nurses & 2 \\
\hline - Medical Assistant & 2 \\
\hline - Community Health Worker & 1 \\
\hline - Director & 1 \\
\hline - Midwife & 1 \\
\hline - $\quad$ PMTCT Program Coordinator & 1 \\
\hline - Psychologist & 1 \\
\hline
\end{tabular}

\subsection{Women's Level}

\subsubsection{Knowledge and Belief in the Potential Effectiveness of the PMTCT Regimen}

Many women indicated that their decision to participate in the PMTCT program was attributed to the effective education provided by HCWs and peer-to-peer interactions that strengthened belief in the program's ability to prevent HIV transmission. Once the women understood that it was possible to give birth to an HIV-negative baby, they felt encouraged and internally motivated to follow the program's guidelines. As one woman described:

"I did not have any sons, so when [HCWs] told me about the program, I told myself that I was going to be able to have another baby...” (PMTCT participant)

In the words of another participant:

"Before, because I knew that I lived with the disease, I could not have children. And when [peers] told me about AED and the advice given there, especially when we heard about the PMTCT program, I agreed. We 
also saw our friends, who had HIV, before us, have children. My husband and I had decided to be part of the program to be supervised in order to be able to have children." (РMTCT participant)

As a HCW stated:

"When the information, that it is to ensure that their children do not become sick, is given well, it motivates them." (HCW)

Furthermore, Togolese radio was identified as an important tool in encouraging HIV-positive pregnant women to seek support and treatment. When one PMTCT participant found out that she was HIV positive during her pregnancy, she was not discouraged. As she stated:

"They often say on the radio that help is available for people [who are HIV positive]." (PMTCT participant)

Through the information broadcasted, participants knew that there was hope despite their status and ultimately came to believe in the promise of the PMTCT program to allow them to have an HIV-negative infant.

\subsubsection{Internal Motivation}

The understanding and belief in the program's goals resulted in women's internal motivation, which was strongly related to adherence to the PMTCT program guidelines. Many women reported that they would ask themselves why they were walking several miles to attend a prenatal appointment, when instead they could be earning money for their family. Others considered discontinuation of their ART because the daily dosage was too taxing. The belief in delivering a healthy, HIV-negative baby, however, explained the continued internal motivation to complete program guidelines despite these competing responsibilities. When one participant was asked if she ever questioned why she had to go to appointments, she stated:

"I can ask myself these questions, and I respond to myself that it is for the health of my baby that I come". (PMTCT participant)

Empowered by their internal motivation to succeed, many women described the problem-solving approaches they then developed to ensure medication adherence. Some used a preset alarm on their cellphones that rang at certain times as a reminder to take their medications. Others remembered to take their regimens when a certain television program aired. Other participants would place their medications in a convenient, yet intentional place (i.e. in purse, under pillow), so that no matter where they were, they would remember to take their medications on time. As one participant stated:

"I cannot forget [to take my medicine] because my phone has a reminder set. Wherever I go, my medications are in my purse. No matter when the time arrives [to take the medications], I take [them].” (PMTCT participant)

When the women were questioned as to why they took these extra steps, participants emphasized again that they wanted to do everything to follow recommendations to ensure a healthy, HIV-negative baby.

\subsection{Supportive Communities}

\subsubsection{Responsive Health Facility and Health Care Workers}

When women felt a personal connection with the HCWs, did not feel stigmatized, and were welcomed and treated well at the facility, they were further motivated to continue program adherence. As one HCW stated:

"When women are welcomed and well cared for in a convivial atmosphere without suspicion, without discrimination, without stigma, they are very motivated to visit you at home and your office. Wherever they see you they ask you things." (HCW)

The connection felt with personnel inspired women to strive towards program adherence and seek out help whenever questions or difficult circumstances arose.

The socio-economic support provided by the respective health facility (AED-Lidaw) also internally motivated and enabled women to continue participation. This support ranged from transportation reimbursements, to complete coverage for medical visits and exams, nutritional support, and free or reduced cost pharmaceuticals. As 
one health care worker remarked:

"What motivates most is the aid that is given to women, considering the fact that most are from poor families. So, we noticed that when there are financial incentives and that their transportation cost was covered, there were many women who responded well to keeping appointments." (HCW)

As one woman stated:

"But if they prescribe you products, [like] when I had my first baby, it is AED that paid for those [products] for me. When you have a problem, they listen well and you do not pay anything for your medications. That alone is enough." (PMTCT participant)

\subsubsection{Peer Support}

Support groups conducted by the health facilities were another important factor. Participants were able to serve as treatment partners beyond meetings, by offering advice, encouragement, and tips to make adherence more manageable. In the words of a PMTCT participant:

"After the [support group] meetings, upon returning, we give each other advice on how to make sure that our babies remain in good health" (PMTCT participant)

The ability to share challenges was also important. When participants heard the problems of others, they realized other PMTCT participants were encountering similar or more difficult circumstances than their own. In the words of a participant:

"[At the support group], they gave us advice and when you go to the group, someone tells her problem and someone else tells their problem, and then you no longer think about your problems." (PMTCT participant)

Ultimately, support groups and the sense of community left women feeling encouraged, empowered, and determined to continue following the PMTCT guidelines. As a mid-wife stated:

"[Support groups] motivate women to adhere to the program. In these groups, trust comes since they say 'so I'm not alone in the situation' and therefore find comfort... also support groups help women break free from fear and worry." (HCW)

\subsubsection{Supportive Family}

Many participants expressed that they were afraid to share their status with relatives, or ask for support due to fear of abandonment and stigmatization. One HCW, who was also a former PMTCT participant noted:

"If the woman announces her status to her family and husband, at least one of her parents or the husband will support her... from fear of discrimination or abandonment, a lot of women do not share their status with their husbands. For the parents, the women often doubt their confidentiality..." (HCW)

When participants overcame the fear of abandonment by family members and chose to inform close relatives, many relatives offered participants resources to address some of the burdens associated with PMTCT program adherence. As a HCW stated:

"For spouses, their participation comes in a variety of forms... participation in cost of services, including prenatal consultations, delivery, testing, and vaccination. There are some, when they have understood the [program's] validity, they accompany their wives to meetings to better understand the doctors' explanations, and they participate in support groups... For family members who are informed about the program, they often participate in encouraging and reminding women to take their medications." (HCW)

Financial support from family members also allowed many women to purchase medications and access transportation in order to attend appointments. As one participant stated:

"If I come [to the hospital] and they give me a prescription for a product, [my husband] gives me money... sometimes he gives me money for a [motorcycle-taxi]." (PMTCT participant)

Some family members were even willing take individuals to appointments, using their own vehicles: 
"My father asks about my health, and sometimes he takes me [to appointments] because he has a motorcycle-taxi." (PMTCT participant)

In addition to financial and logistical support, family members also offered emotional support. As a HCW remarked:

"Sometimes, parents and spouses support the women morally and financially, even by reminding them about appointments. For spouses who accompany their wives to support groups, they learn how to help their wives..." (HCW)

One participant remarked about the emotional support from her mother and sister that allowed her to continue taking her medications:

"When I was sick, it was [my mother and sister] who supported me! It was them who have even encouraged me to go to AED." (PMTCT participant)

For another participant, it was the support offered from her partner that contributed to her success:

"Sometimes it is the baby's father who asks me if I took [the medicine]. It is him who reminds me." (PMTCT participant)

\subsubsection{Supportive Faith-Based Community}

Religious gatherings allowed these women to stay connected to a continual, positive stream of support throughout the duration of their PMTCT program participation. Religious leaders offered support to women through prayer, encouragement, and accompaniment to medical visits. As a doctor from CHU-Kara pointed out:

"The women confide in pastors and in priests who accompany them [to appointments] and who comfort them. Often in support groups, they talk about how the priest or pastor helped them to accept their status." (HCW)

The churches were also actively engaged in the health care setting, and some women found support through prayer meetings that were held at the hospital by churches. As a health care worker stated:

"There are some churches that have respondents in the hospitals. For example, each Tuesday, the Catholic Church unites a certain number of people for a special hospital mass, for the personnel and the sick that are there." (HCW)

\section{Discussion}

We were able to identify critical patient and HCW-reported factors that facilitated women's successful participation in and adherence to PMTCT programs in Kara, Togo. The driving force was women's knowledge of and belief in the efficacy of PMTCT programs in allowing them to have an HIV-negative child. However, for this motivation to result in successful adherence, support from a range of communities was also needed to reinforce their commitment and help them to overcome structural barriers.

Overall, our findings are consistent with other studies, suggesting the importance of effective education on the PMTCT program, internal motivation, a responsive health facility, supportive peers and relatives [13]-[18].

HCWs were critical to helping build the motivation and resulting empowerment for women to adhere to the PMTCT program, through effective education regarding the efficacy of PMTCT. Nyasula and others found that shortage of HCWs in Malawi and other settings leads to inadequate PMTCT program education and inaccurate assessments of participants' situations [19] [20]. Ensuring that there are HCWs knowledgeable about and trained to deliver effective education as a core component of antenatal and HIV services will be important to achieving the patient motivation related to a belief in the promise of PMTCT. Increasing women's knowledge was supported by other sources including radio broadcasts, which provided public service announcements highlighting the help available for HIV-positive individuals. Kuhlmann and Lovell found that media outlets could have a positive impact on decreasing MTCT and HIV transmission in Botswana [21] [22]. Future studies should further explore the most effective ways for media outlets to further increase PMTCT program participation and adherence.

While knowledge and education was crucial to initial participation in the PMTCT, internal motivation from 
the resulting belief in the promise of a healthy baby led to continued adherence. Findings from earlier studies found that a lack of internal motivation contributed to inadequate antiretroviral uptake and subsequently, poor PMTCT adherence [23] [24]. Empowered by this internal motivation to succeed, a women's ability to problem solve and adapt her lifestyle allowed her to find ways through which she could continue to participate, reflecting a determination to adhere regardless of obstacles. A focus on instilling and supporting this empowerment through initial PMTCT education and problem-solving skill building is another important approach to enable women to successfully adhere to the PMTCT program.

However knowledge and motivation were only effective when supported by three communities: the health care system, families and peers, and for some women, their religious community. The World Health Organization defines a responsive health facility as one able to effectively respond to the expectations of the population it treats [25]. Sprague et al. in South Africa found that non-responsive health facilities, weak in services offered and support provided, lead to inadequate PMTCT participation and adherence [26]. Our findings reinforce the importance of a strong, supportive health facility that welcomes and comforts participants, while providing them with resources necessary for program adherence. Building a peer community by offering the opportunity for peer-to-peer support was also an important factor for success. Other studies have shown the positive impact of facility-based "Mother-to-Mother" support groups [6] [27]. Shroufi et al. in Zimbabwe found that psychosocial support empowers HIV positive, pregnant women to enact positive behavioral changes through the information gained from peer-to-peer interactions [2].

Male partner involvement is an important factor to increase PMTCT program adherence; but is difficult to achieve [28] [29]. Engaging other influential relatives and friends, through educational efforts and medical appointments, may provide alternate and additional support outlets for women, further reinforcing the internal motivation leading to PMTCT success [30] [31]. Ware et al. described the powerful role of non-spousal individuals in improving ART adherence within Nigeria, Tanzania, and Uganda [3]. O'Laughlin et al. in Tanzania also found improved ART adherence for individuals who had treatment support partners [32]. Since many women were able to rely on the support of non-spousal relatives, it is important to educate and integrate family members and other members of a woman's support network on PMTCT so they can provide the additional reinforcement of adherence.

The role of faith-based organizations in ART uptake and adherence has been mixed. Campbell and Keikelame noted that faith-based organizations can inhibit or facilitate HIV prevention measures [33] [34]. In contrast, our findings suggest that welcoming faith-based communities and leaders provides additional support and encouragement for PMTCT participants. More research is needed on how to better engage religious leaders and faith-based communities to support women in PMTCT program uptake and adherence.

\subsection{Limitations}

Our study had a number of limitations. Interviews were analyzed and coded by one individual, who had previous training and knowledge of qualitative research, fluent in Togolese-spoken French, and knowledge of the Togolese culture. This approach was chosen to reduce the risk of loss of meaning, particularly in Togolese spoken French and Togolese culture [35]. While it is possible that additional themes may have emerged, a random sample of ten out of forty-five transcripts was analyzed and coded by a second researcher fluent in French and also familiar with Togolese culture; no additional themes were identified. Furthermore, the lack of data available on PMTCT women who did not complete the program limits the extent to which we could compare information on successful versus unsuccessful participants. Additionally, social desirability bias could have resulted in women over reporting positive instances of factors that enabled success, particularly as we interviewed them within the health facility [36]. To reduce bias, interviewees were reassured that the interview results would remain anonymous and questions were worded and posed in a neutral manner [37]. Furthermore, recall bias may have prevented women from fully expressing various events that facilitated continued PMTCT participation [38] [39]. In order to reduce this bias, participants were asked a series of time-line specific questions that stimulated their memory on events surrounding their PMTCT participation [40]. Importantly, many of the women had not completed the full 18-month post-partum PMTCT follow-up guidelines, so these results are primarily relevant to the first two of the three components of effective PMTCT (antenatal and peri-partum). Most (80\%) of the women had prior knowledge of their HIV status and were on treatment before becoming pregnant, so the findings may not be as generalizable to women who are diagnosed during pregnancy. Finally, because the study only included 
participants from two sites within the same region, findings may have limited generalizability to other regions.

\subsection{Conclusions}

Capturing the voice of women successful in completing a PMTCT program and the HCWs who helped facilitate this success can identify important areas for supporting other women to achieve similar goals. Ensuring that the women have the knowledge of the potential to have an HIV-negative baby, and the support to build on resulting internal motivation driven by a desire and belief in having an HIV-negative child represents a package, which can help strengthen the effectiveness of expanding PMTCT programs. Key areas where work is needed include training of HCWs to deliver effective patient education, active development of support communities for women and a welcoming and responsive healthcare environment. Work to further strengthen the critical role of an accepting community outside of the family and the clinic, through public media and other public service messaging strategies as well as through faith-based communities, is also critical-highlighting the need to reach beyond traditional health systems to achieve the goal of an AIDS-free generation.

\section{Acknowledgements}

The authors wish to thank: study participants for their willingness to share their stories and experiences; The United States Department of State Fulbright Student Program Scholarship for project funding; and, Annie Michaelis, Andrew Lopez, and Kristefer Stanjovanski for excellent technical support.

\section{Disclosures}

None.

\section{References}

[1] Tolle, M. and Dewey, D. (2010) Prevention of Mother-to-Child Transmission of HIV Infection. In: Baylor College of Medicine, Ed., HIV Curriculum for the Health Professional, Baylor International Pediatric AIDS Initiative, Houston, 90-119.

[2] Shroufi, A., Mafara, E., Saint-Sauveur, J.F., Taziwa, F. and Vinoles, M.C. (2013) Mother to Mother (M2M) Peer Support for Women in Prevention of Mother to Child Transmission (PMTCT) Programmes: A Qualitative Study. PLoS One, 8, e64717. http://dx.doi.org/10.1371/journal.pone.0064717

[3] Ware, N.C., Wyatt, M.A. and Bangsberg, D.R. (2006) Examining Theoretic Models of Adherence for Validity in Resource-Limited Settings. A Heuristic Approach. Journal of Acquired Immune Deficiency Syndromes, 43, S18-S22. http://dx.doi.org/10.1097/01.qai.0000248343.13062.4b

[4] Direction Regionale de la Sante (DRS), Region de la Kara (2011) Rapport des Activites Sanitaires de la Region de la Kara 2011. DRS, Lome, 43-46.

[5] Bwirire, L.D., et al. (2008) Reasons for Loss to Follow-Up among Mothers Registered in a Prevention-of-Mother-toChild Transmission Program in Rural Malawi. Transactions of the Royal Society of Tropical Medicine and Hygiene, 102, 1195-1200. http://dx.doi.org/10.1016/j.trstmh.2008.04.002

[6] Viadro, C. (2008) Mothers' Support Groups in Ethiopia: A Peer Support Model to Address the Needs of Women Living with HIV. Intra Health.

[7] Bradley, E.H., Curry, L.A., Ramanadhan, S., Rowe, L., Nembhard, I.M. and Krumholz, H.M. (2009) Research in Action: Using Positive Deviance to Improve Quality of Health Care. Implement Science, 4, 25. http://dx.doi.org/10.1186/1748-5908-4-25

[8] Hope through Health. Prevention of Mother to Child Transmission of HIV. www.hthglobal.org

[9] World Health Organization (2010) Antiretroviral Drugs for Treating Pregnant Women and Preventing Infection in Infants: Recommendations for a Public Health Approach. http://www.who.int/en/

[10] The World Health Organization (2010) New Guidance on Prevention of Mother-to-Child Transmission of HIV and Infant Feeding in the Context of HIV. http://www.who.int/hiv/pub/mtct/PMTCTfactsheet/en/

[11] Suter, W. (2012) Qualitative Data, Analysis, and Design. In: Newton Suter, W., Ed., Introduction to Educational Research: A Critical Thinking Approach, Sage Publications, Thousand Oaks, 342-386.

[12] Berg, B. (2007) An Introduction to Content Analysis. In: Berg, B.L., Ed., Qualitative Research Methods for the Social Sciences, Allyn and Bacon, Boston, 238-267. 
[13] Kumarasamy, N., Safren, S.A., Raminani, S.R., Pickard, R., James, R., Krishnan, A.K., et al. (2005) Barriers and Facilitators to Antiretroviral Medication Adherence among Patients with HIV in Chennai, India: A Qualitative Study. AIDS Patient Care STDS, 19, 526-537. http://dx.doi.org/10.1089/apc.2005.19.526

[14] Matthews, L.T., Crankshaw, T., Giddy, J., Kaida, A., Psaros, C., Ware, N.C., et al. (2012) Reproductive Counseling by Clinic Healthcare Workers in Durban, South Africa: Perspectives from HIV-Infected Men and Women Reporting Serodiscordant Partners. Infectious Diseases in Obstetrics and Gynecology, 2012, Article ID: 146348.

[15] Frank, J. and Duncan, N. (2009) Speaking to Experts and Patients: Recommendations for Improving Antiretroviral Therapy (ART) Adherence. Health SA Gesondheid, 14, 9 p.

[16] Chinkonde, J.R., Sundby, J. and Martinson, F. (2009) The Prevention of Mother-to-Child HIV Transmission Programme in Lilongwe, Malawi: Why Do So Many Women Drop Out. Reproductive Health Matters, 17, $143-151$. http://dx.doi.org/10.1016/S0968-8080(09)33440-0

[17] Muchedzi, A., Chandisarewa, W., Keatinge, J., Stranix-Chibanda, L., Woelk, G., Mbizvo, E. and Shetty, A.K. (2010) Factors Associated with Access to HIV Care and Treatment in a Prevention of Mother to Child Transmission Programme in Urban Zimbabwe. Journal of the International AIDS Society, 13, 38. http://dx.doi.org/10.1186/1758-2652-13-38

[18] Otolok-Tanga, E., Atuyambe, L., Murphy, C.K., Ringheim, K.E. and Woldehanna, S. (2007) Examining the Actions of Faith-Based Organizations and Their Influence on HIV/AIDS-Related Stigma: A Case Study of Uganda. African Health Sciences, 7, 55-60.

[19] Nyasulu, J.Y. and Nyasulu, P. (2011) Barriers to the Uptake of Prevention of Mother to Child Transmission (PMTCT) Services in Rural Blantyre and Balaka Districts, Malawi. Journal of Rural and Tropical Public Health, 10, 48-52.

[20] Adedimeji, A., Abboud, N., Merdekios, B. and Shiferaw, M. (2012) A Qualitative Study of Barriers to Effectiveness of Interventions to Prevent Mother-to-Child Transmission of HIV in Arba Minch, Ethiopia. International Journal of Population Research, 2012, 7.

[21] Sebert Kuhlmann, A.K., Kraft, J.M., Galavotti, C., Creek, T.L., Mooki, M. and Ntumy, R. (2008) Radio Role Models for the Prevention of Mother-to-Child Transmission of HIV and HIV Testing among Pregnant Women in Botswana. Health Promotion International, 23, 260-268. http://dx.doi.org/10.1093/heapro/dan011

[22] Lovell, C.C., Pappas-DeLuca, K.A., Sebert Kuhlmann, A.K., Koppenhaver, T., Kong, S., Mooki, M. and Galavotti, C. (2007) “One Day I Might Find Myself HIV-Positive like Her”: Audience Involvement and Identification with Role Models in an Entertainment-Education Radio Drama in Botswana. International Quarterly of Community Health Education, 28, 181-200. http://dx.doi.org/10.2190/IQ.28.3.b

[23] Houston, E., McKirnan, D.J., Cervone, D., Johnson, M.S. and Sandfort, T.G.M. (2011) Assessing Treatment Motivation among Patients Receiving Antiretroviral Therapy: A Multidimensional Approach. Psychology \& Health, 27, 674687.

[24] Holstad, M.M., Dilorio, C. and Magowe, M.K. (2006) Motivating HIV Positive Women to Adhere to Antiretroviral Therapy and Risk Reduction Behavior: The KHARMA Project. The Online Journal of Issues in Nursing, 11, 5.

[25] World Health Organization (2014) Health Systems. http://www.who.int/trade/glossary/story049/en/

[26] Sprague, C., Chersich, M.F. and Black, V. (2011) Health System Weaknesses Constrain Access to PMTCT and Maternal HIV Services in South Africa: A Qualitative Enquiry. AIDS Research and Therapy, 8, 10. http://dx.doi.org/10.1186/1742-6405-8-10

[27] Khan, H. (2007) From Mother to Mother: A Peer Mentor Program to Prevent Mother-to-Child Transmission of HIV in South Africa Offers Much Needed Support.

[28] Nsagha, D.S., Halle-Ekane, G.E., Nfor, C.S., Ngowe, M.N. and Nasah, B.T. (2014) The Role of the Male Partner in the Prevention of Mother to Child Transmission of HIV in Cameroon. American Journal of Epidemiology and Infectious Disease, 2, 52-59. http://dx.doi.org/10.12691/ajeid-2-2-1

[29] Kalembo, F.W., Zgambo, M., Mulaga, A.N., Yukai, D. and Ahmed, N.I. (2013) Association between Male Partner Involvement and the Uptake of Prevention of Mother-to-Child Transmission of HIV (PMTCT) Interventions in Mwanza District, Malawi: A Retrospective Cohort Study. PLoS ONE, 8, e66517. http://dx.doi.org/10.1371/journal.pone.0066517

[30] Knodel, J., Kespichayawattana, J., Saengtienchai, C. and Wiwatwanich, S. (2010) The Role of Parents and Family Members in ART Treatment Adherence: Evidence from Thailand. Research on Aging, 32, 19-39. http://dx.doi.org/10.1177/0164027509348130

[31] Binagwaho, A. and Ratnayake, N. (2009) The Role of Social Capital in Successful Adherence to Antiretroviral Therapy in Africa. PLoS Medicine, 6, e18. http://dx.doi.org/10.1371/journal.pmed.1000018

[32] O’Laughlin, K.N., Wyatt, M.A., Kaaya, S., Bangsberg, D.R. and Ware, N.C. (2012) How Treatment Partners Help: Social Analysis of an African Adherence Support Intervention. AIDS and Behavior, 16, 1308-1315. 
http://dx.doi.org/10.1007/s10461-011-0038-4

[33] Campbell, C., Skovdal, M. and Gibbs, A. (2011) Creating Social Spaces to Tackle AIDS-Related Stigma: Reviewing the Role of Church Groups in Sub-Saharan Africa. AIDS and Behavior, 15, 1204-1219. http://dx.doi.org/10.1007/s10461-010-9766-0

[34] Keikelame, M.J., Murphy, C.K., Ringheim, K.E. and Woldehanna, S. (2010) Perceptions of HIV/AIDS Leaders about Faith-Based Organizations’ Influence on HIV/AIDS Stigma in South Africa. African Journal of AIDS Research, 9, 6370. http://dx.doi.org/10.2989/16085906.2010.484571

[35] van Nes, F., Abma, T., Jonsson, H. and Deeg, D. (2010) Language Differences in Qualitative Research: Is Meaning Lost in Translation? European Journal of Ageing, 7, 313-316. http://dx.doi.org/10.1007/s10433-010-0168-y

[36] Hardon, A, Vernooij, E., Bongololo-Mbera, G., Cherutich, P., Desclaux, A., Kyaddondo, D., et al. (2012) Women’s Views on Consent, Counseling and Confidentiality in PMTCT: A Mixed-Methods Study in Four African Countries. BMC Public Health, 12, 26. http://dx.doi.org/10.1186/1471-2458-12-26

[37] Nederhof, A. (1985) Methods of Coping with Social Desirability Bias: A Review. European Journal of Social Psychology, 15, 263-280. http://dx.doi.org/10.1002/ejsp.2420150303

[38] Wiegert, K., Dinh, T.H., Mushavi, A., Mugurungi, O. and Kilmarx, P.H. (2014) Integration of Prevention of Mother-to-Child Transmission of HIV (PMTCT) Postpartum Services with Other HIV Care and Treatment Services within the Maternal and Child Health Setting in Zimbabwe, 2012. PLoS ONE, 9, e98236. http://dx.doi.org/10.1371/journal.pone.0098236

[39] Mnyani, C.N. and McIntyre, J. (2013) Challenges to Delivering Quality Care in a Prevention of Mother-to-Child Transmission of HIV Programme in Soweto, South Africa. Southern African Journal of HIV Medicine, 14.

[40] Rothman, K.J. (2002) Epidemiology: An Introduction. Oxford University Press, New York. 
Scientific Research Publishing (SCIRP) is one of the largest Open Access journal publishers. It is currently publishing more than 200 open access, online, peer-reviewed journals covering a wide range of academic disciplines. SCIRP serves the worldwide academic communities and contributes to the progress and application of science with its publication.

Other selected journals from SCIRP are listed as below. Submit your manuscript to us via either submit@scirp.org or Online Submission Portal.
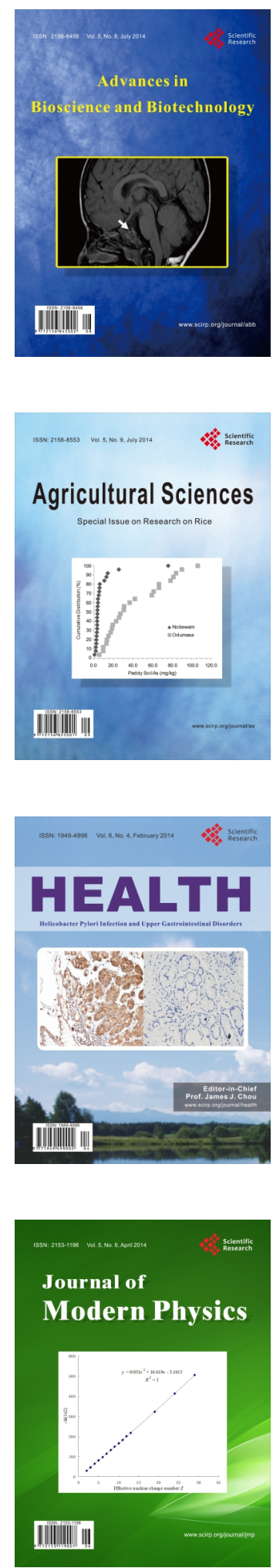
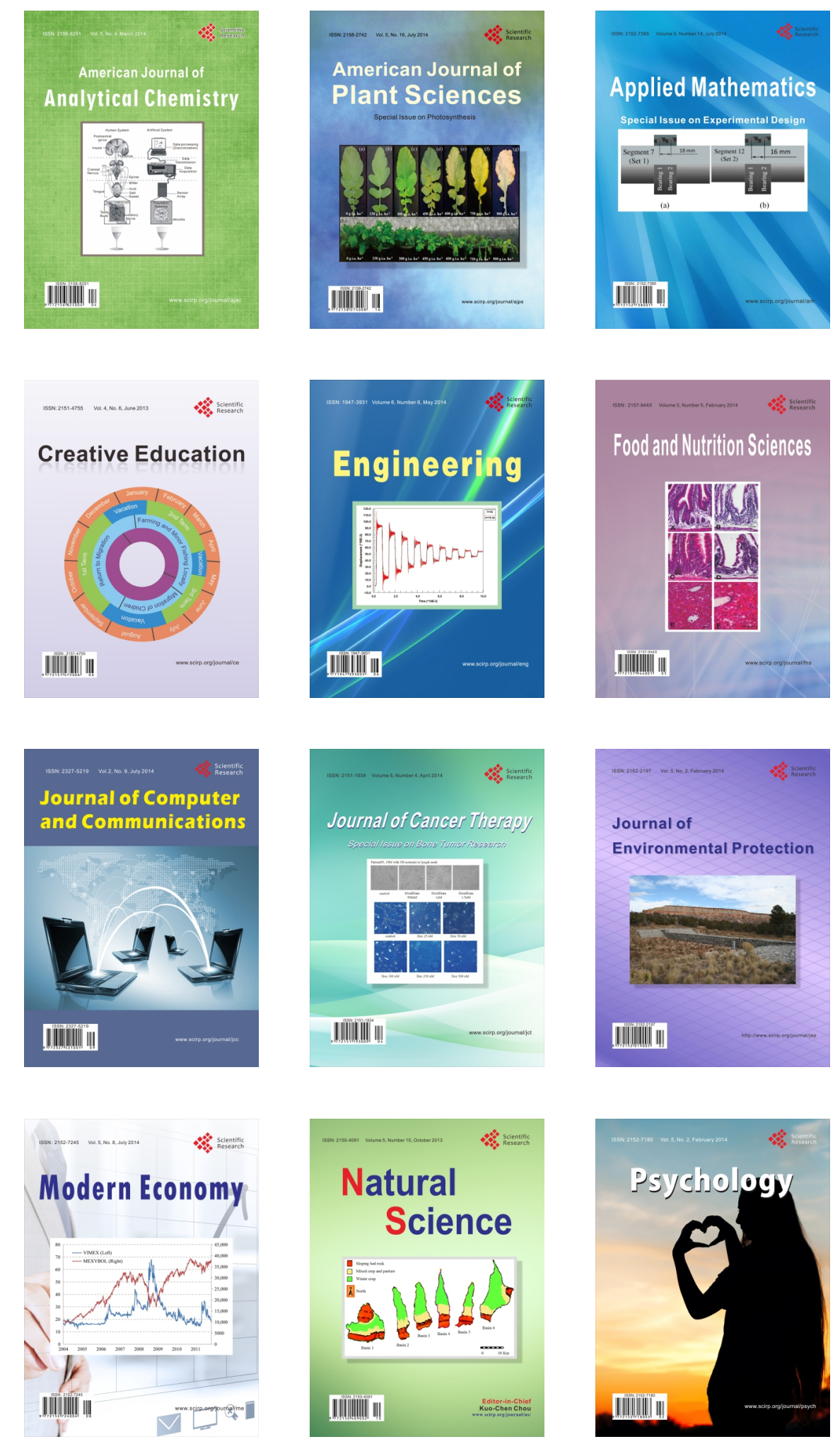